\title{
Multibranch pulse synthesis and electro-optic detection of subcycle multi-terahertz electric fields
}

\author{
Philipp Merkl, Matthias Knorr, Christian Meineke, lukas Kastner, Dominik Peller, \\ and RUPert Huber*
}

Department of Physics, University of Regensburg, 93040 Regensburg, Germany

*Corresponding author: rupert.huber@ur.de

Received 30 August 2019; revised 21 October 2019; accepted 24 October 2019; posted 24 October 2019 (Doc. ID 376731); published 8 November 2019

\begin{abstract}
We present a robust, compact pulse synthesis scheme generating intense phase-locked subcycle multi-terahertz waveforms. The ultrabroadband laser fundamental is split into two parallel branches driving optical rectification in crystals of $\mathrm{GaSe}$ and $\mathrm{LiGaS}_{2}$, each operated at the group velocity matching point. The coherent combination of the resulting pulses yields a continuous multi-terahertz spectrum covering 1.5 optical octaves. The corresponding 0.8 -cycle electric field waveform is directly mapped out by electro-optic sampling, revealing peak fields of $15 \mathrm{kV} / \mathrm{cm}$ at a repetition rate of $0.4 \mathrm{MHz}$. The multiplexable and power scalable scheme opens the door to strong-field custom-tailored waveforms driving nonlinear optics and light wave electronics. () 2019 Optical Society of America
\end{abstract}

https://doi.org/10.1364/OL.44.005521

Ultrashort light pulses in the terahertz (THz) and mid-infrared (MIR) spectral range are unique probes of low-energy excitations in condensed matter [1,2]. The combination of carrierenvelope phase (CEP) stable waveforms with electro-optic sampling [3-8] (EOS) has been used to track nonequilibrium dynamics, on subcycle time scales $[1,2]$. At sufficiently high intensities [9-14], THz light wave electronics [10-14] have become possible, where the $\mathrm{THz}$ carrier wave serves as an atomically strong bias field to accelerate electrons [11-13], drive Bloch oscillations [11], and generate high harmonics in bulk crystals $[11,12]$. Recently, light-wave-driven scanning tunneling microscopy (STM) [15-18] has introduced a spectacular paradigm by imaging the motion of a single molecular orbital with a $100 \mathrm{fs}$ resolution [17]. For this purpose, a CEP-stable subcycle $\mathrm{THz}$ wave has been coupled into the tip-sample tunneling junction as a transient bias that opens a resonant singleelectron tunneling channel through a select molecular orbital during a fraction of the most intense $\mathrm{THz}$ half-cycle. Extending the carrier frequency from the few- $\mathrm{THz}$ to the multi-THz/MIR spectral range may shorten this tunneling time window and hence improve the temporal resolution to a few femtoseconds, bringing even slow-motion movies of electronic dynamics into reach.

All these applications call for a challenging combination of large bandwidth, field strength, and repetition rate. For the example of light-wave-driven STM, subcycle waveforms imply octavespanning bandwidths, while single-electron tunneling requires repetition rates of hundreds of kilohertz $(\mathrm{kHz})$ to provide measurable currents. Recent approaches, reaching the single-cycle regime with large peak fields, have used optical rectification of femtosecond near-infrared (NIR) pulses under group velocity matching conditions [19,20], filamentation in gases [21], pulse self-compression [22], or adiabatic difference frequency mixing [23], mostly at $\mathrm{kHz}$ repetition rates. Another promising concept to simultaneously reach high conversion efficiencies, octavespanning bandwidths, and high repetition rates may exploit coherent pulse synthesis [24,25]. In a MIR optical parametric amplifier, a signal and idler combined spectrum has spanned the window from 2.5 to $9.0 \mu \mathrm{m}$ [26]. The concept of coherent pulse synthesis of MIR transients has not been extended into the multi- $\mathrm{THz}$ range above $10 \mu \mathrm{m}$ yet, and a full electro-optic analysis of pulses synthesized this way has not been reported.

Here, we introduce a robust, multiplexable, and powerscalable pulse synthesis scheme based on optical rectification, generating passively CEP-stable 0.8 -cycle multi-THz fields up to $15 \mathrm{kV} / \mathrm{cm}$ at a scalable repetition rate of $0.4 \mathrm{MHz}$. The spectrum extends from 11 to $30 \mathrm{THz}$ (FWHM) corresponding to 1.5 optical octaves, while EOS allows for a direct and full characterization of the generated subcycle waveform.

Our concept resolves a key dilemma of ultrabroadband optical rectification of femtosecond laser pulses: efficient frequency conversion requires long crystal lengths, $L$, and yet phase matching can only be fulfilled in a finite spectral window whose width shrinks with increasing $L$. The largest possible bandwidth is achieved when not only the phase, but also the group velocities are matched [19]. For a given nonlinear optical medium, this condition can typically be satisfied for only one specific crystal angle and, thus, only one $\mathrm{THz}$ center frequency. By combining complementary spectra obtained from different nonlinear optical materials, each operated in 
their group velocity matched geometries, one may expect particularly broadband spectra.

The schematic is sketched in Fig. 1. For our demonstration experiment, we employ a home-built Ti:sapphire laser amplifier system, providing $20 \mathrm{fs}$ pulses (center wavelength, $\lambda_{p}=790 \mathrm{~nm}$; bandwidth, $\Delta \lambda=60 \mathrm{~nm}$; pulse energy, $0.75 \mu \mathrm{J})$ at a repetition rate of $0.4 \mathrm{MHz}$. A beam splitter transmits $90 \%$ of the output power into the pulse synthesis scheme. Subsequently, the beam is split into two branches with tunable intensity, using a combination of a half-wave plate and a polarizing beam splitter cube. A fused silica lens (focal length, $10 \mathrm{~cm}$ ) focuses the two branches into the nonlinear optical crystals with peak intensities of $\sim 0.2 \mathrm{TW} / \mathrm{cm}^{2}$. Two adjustable fused silica wedges allow for fine tuning of the time delay between both branches, while a fused silica plate of a thickness of $3 \mathrm{~mm}$ balances the dispersion in both branches. The multi-THz waveforms are generated by optical rectification of the NIR laser pulses in a $\mathrm{LiGaS}_{2}$ (LGS, thickness, $L_{\mathrm{LGS}}=300 \mu \mathrm{m}$ ) and a GaSe (thickness, $L_{\mathrm{GaSe}}=70 \mu \mathrm{m}$ ) crystal, simultaneously. Thereby, the short- and long-wavelength wings of the laser spectrum serve as pump and signal waves in an intrapulse difference frequency generation (DFG) process yielding $\mathrm{THz}$ idler waves.

Efficient conversion requires the momentum mismatch, $\Delta k$, between the pump, signal, and idler photons to nearly vanish ${ }^{19}$. Figures 2(a) and 2(b) depict the corresponding phase-matching function $|\operatorname{sinc}(\Delta k L / 2)|$ for the two nonlinear crystals in dependence of the internal phase-matching angle, $\theta$, and the frequency of the $\mathrm{THz}$ wave, $\nu_{\mathrm{THz}}$. The maximum emission bandwidth occurs at the stationary points of perfect group velocity matching at $\theta_{\mathrm{GaSe}}=13^{\circ}$ and $\theta_{\mathrm{LGS}}=49^{\circ}$ [Figs. 2(a) and 2 (b), horizontal dashed lines]. There, the phase-matching

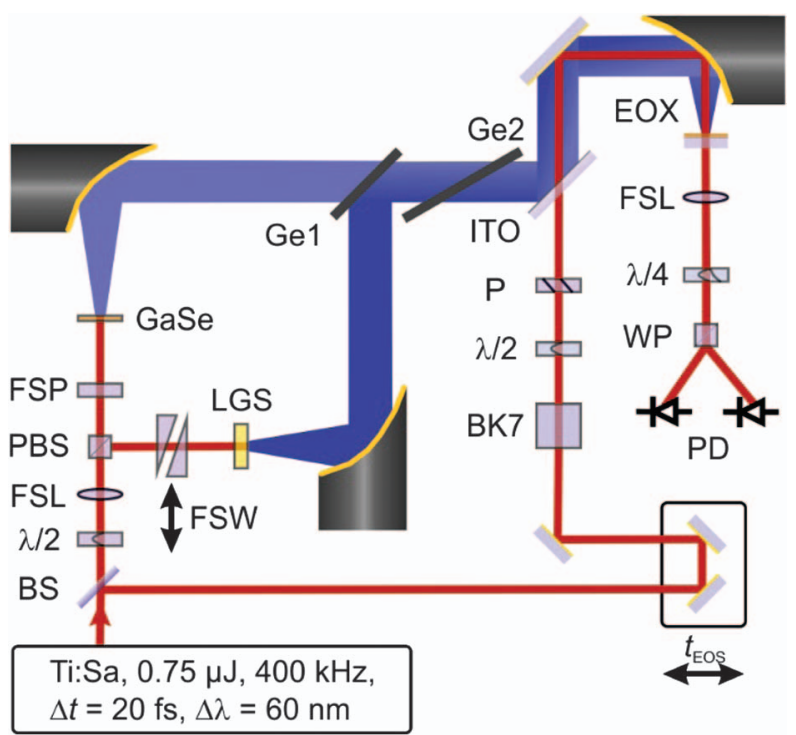

Fig. 1. Schematic for subcycle multi-THz waveform synthesis and electro-optic detection. BS, beam splitter $(T=90 \%) ; \lambda / 2$, half-wave plate; FSL, fused silica lens $(f=10 \mathrm{~cm})$; PBS, polarizing beam splitter cube; FSP, fused silica plate; GaSe, $70 \mu \mathrm{m}$ GaSe crystal; FSW, fused silica wedges; LGS, $300 \mu \mathrm{m}$ LGS crystal; Ge1, $500 \mu \mathrm{m}$ Ge beam combiner; Ge2, Ge NIR beam block; BK7, 10 mm BK7 plate; P, thin-film polarizer; ITO, fused silica ITO window; EOX, $5 \mu \mathrm{m}$ GaSe contacted on diamond window; $\lambda / 4$, quarter-wave plate; WP, Wollaston prism; $\mathrm{PD}$, balanced pair of photodiodes.
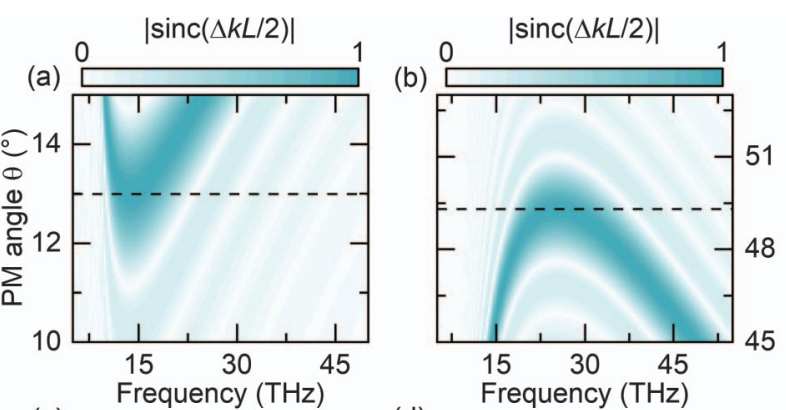

(c)

(d)
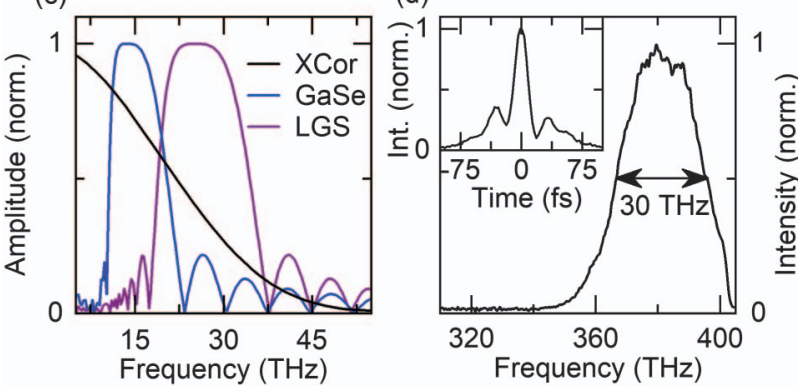

Fig. 2. Phase matching (PM) for optical rectification in $\mathrm{GaSe}$ and LGS. (a) PM function $|\operatorname{sinc}(\Delta k L / 2)|$ (color coded) as function of the PM angle $\theta$ and the idler frequency for type-II DFG in a $70 \mu \mathrm{m}$ thick GaSe crystal and (b) type-I DFG in a $300 \mu \mathrm{m}$ thick LGS crystal for pump wavelength $\lambda_{p}=790 \mathrm{~nm}$. The PM angle given here corresponds to the internal propagation angle relative to the $Z$ axis in GaSe, and relative to the $X$ axis in LGS. Black dashed lines indicate cross sections shown in (c). (c) PM curves for perfect group velocity matching for GaSe (blue curve) and LGS (purple curve) and spectrum of intrapulse difference frequencies within the NIR laser pulse (XCor, black curve). (d) Intensity spectrum of the NIR-driving field and intensity autocorrelation (inset).

bandwidths (FWHM) extend from 10 to $20 \mathrm{THz}$ [Fig. 2(c), blue curve] and 18 to $34 \mathrm{THz}$ [Fig. 2(c), purple curve] for GaSe and LGS, respectively.

The range of actually generated idler frequencies also depends on the bandwidth of the incident laser pulse [Fig. 2(d)]. The spectrum of possible intrapulse difference frequencies in the driving NIR pulse is indicated by the black line in Fig. 2(c). By numerically solving the nonlinear wave equations, including the pump spectrum and the phase-matching curves, and subsequent superposition of the resulting spectra, we find that bandwidths of up to 1.5 optical octaves may be possible.

In the experiment, we employ a $500 \mu \mathrm{m}$ thick Ge-wafer (Ge1) under an angle of $45^{\circ}$ to coherently combine the multi$\mathrm{THz}$ pulses generated in both branches. A second $1 \mathrm{~mm}$ thick Ge-wafer (Ge2) placed under Brewsters angle removes residual fundamental laser light and compensates for the group delay dispersion introduced by the nonlinear crystals.

We directly trace the electric field of the multi-THz pulses by EOS. To this end, $10 \%$ of the laser output is reflected off a beam splitter to serve as the gate [pulse duration, $20 \mathrm{fs}$; see inset in Fig. 2(d)]. After a variable delay line $\left(t_{\mathrm{EOS}}\right)$, the gate passes through a BK7 window of a thickness of $10 \mathrm{~mm}$ to compensate residual chirp. The gate is temporally overlapped with the multi$\mathrm{THz}$ pulses by transmission through a fused silica window coated with indium-tin oxide (ITO). Subsequently the multi- $\mathrm{THz}$ waveform and the NIR gate are collinearly focused into a 
$5 \mu \mathrm{m}$ thick GaSe crystal contacted on a $500 \mu \mathrm{m}$ diamond substrate. The van-der-Waals contacted detector crystal provides an etalon-free electro-optic response up to $100 \mathrm{THz}$ [8]. By analyzing the change in polarization of the gate pulse with a quarterwave plate, a Wollaston prism, and a balanced pair of photo diodes, the full electric field waveform of the multi-THz pulses is retrieved.

Figure 3 depicts the $\mathrm{THz}$ transients from the two individual branches. In both cases, the existence of well-defined waveforms clearly proves the CEP stability of the THz pulse trains. The transients generated in GaSe and LGS [Figs. 3(a) and 3(c), black waveforms] both feature a pulse duration of only 48 fs (intensity FWHM), close to the single-cycle limit. The corresponding spectral amplitudes and absolute phases are shown in Figs. 3(b) and $3(\mathrm{~d})$. The waveform generated in GaSe is centered at a frequency of $19 \mathrm{THz}$ and nearly transform limited with a phase variation of less than $\pi / 4$, over the entire spectral FWHM between 11 and $22 \mathrm{THz}$. The spectral amplitude of the transient generated in LGS is centered at $27 \mathrm{THz}$ with a width of $13 \mathrm{THz}$ (FWHM), while the phase shows a minor residual chirp of $350 \mathrm{fs}^{2}$ caused by the material dispersion of the nonlinear medium. Given the complementary spectral coverage, coherent superposition of the electric field waveforms should further reduce the pulse duration into the subcycle regime. (a)

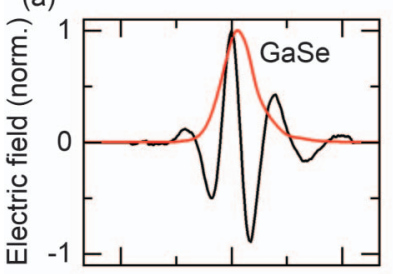

(c)

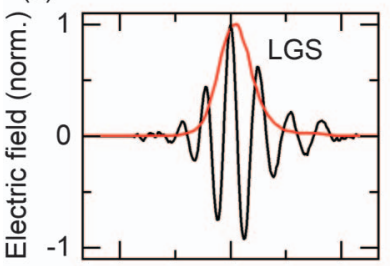

(e)

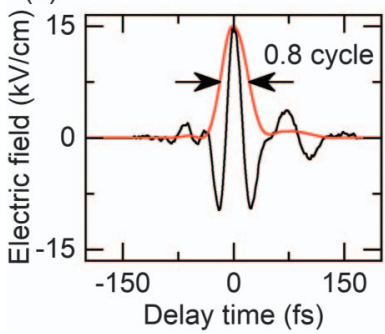

(b)

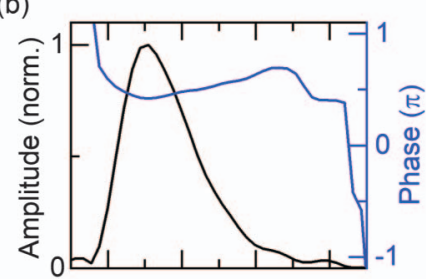

(d)

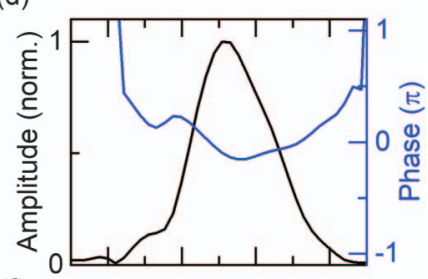

(f)

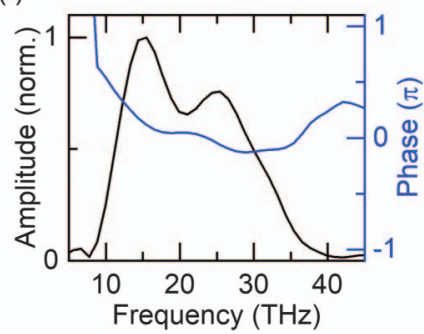

Fig. 3. Phase-locked multi-THz transients and synthesized subcycle waveform. Waveforms generated in a (a) $70 \mu \mathrm{m} \mathrm{GaSe}$ and a (c) $300 \mu \mathrm{m}$ LGS crystal retrieved by electro-optic detection. (e) Waveform (black curve) synthesized by temporally overlapping the waveforms shown in (a) and (c). The pulse features 0.8 cycles within the FWHM of the intensity envelope (red curve). All field transients have been corrected for the electro-optic detector response. (b), (d), (f) Normalized spectral amplitude (black curves) and phase (blue curves) obtained by Fourier transforming the above waveforms, where (b), (d), and (f) correspond to (a), (c), and (e) respectively.
To this end, we unblock both branches and electro-optically detect the superposition of the two waveforms. In order to minimize the pulse duration, we tune the mutual timing and the relative field strengths of the two waveforms by controlling the position of the fused silica wedges and rotating the first half-wave plate (Fig. 1), respectively. The resulting subcycle electric field waveform is shown in Fig 3(e). Again, the existence of a well-defined waveform directly proves the CEP stability of the synthesized multi-THz pulse. The waveform features a pulse duration of $38 \mathrm{fs}$ at a center frequency of $21 \mathrm{THz}$ yielding 0.8 cycles under the intensity envelope. The pulse energy amounts to $1.5 \mathrm{pJ}$, resulting in a peak field of $15 \mathrm{kV} / \mathrm{cm}$ at a focal spot of $100 \mu \mathrm{m}$. The spectral amplitude [Fig. 3(f), black curve] shows only a minor dip at the overlap region of the two constituting spectra, which extend from 11 to $30 \mathrm{THz}$, covering 1.5 optical octaves. Since the input pulses imprint their spectral phases on the synthesized pulse, the absolute phase varies by less than $\pi / 4$ [Fig. 3(f), blue curve]. Due to the long wavelength of the superimposed field transients and the compact geometry of the scheme, phase jitter effects are negligible compared to coherent pulse synthesis in the NIR and visible spectral domain.

Even though the 0.8 -cycle multi-THz pulse covers 1.5 optical octaves, it does not exploit the full bandwidth of the NIR laser pulse because the generation of low- $\mathrm{THz}$ frequency components is limited to above $8 \mathrm{THz}$ by the reststrahlen band
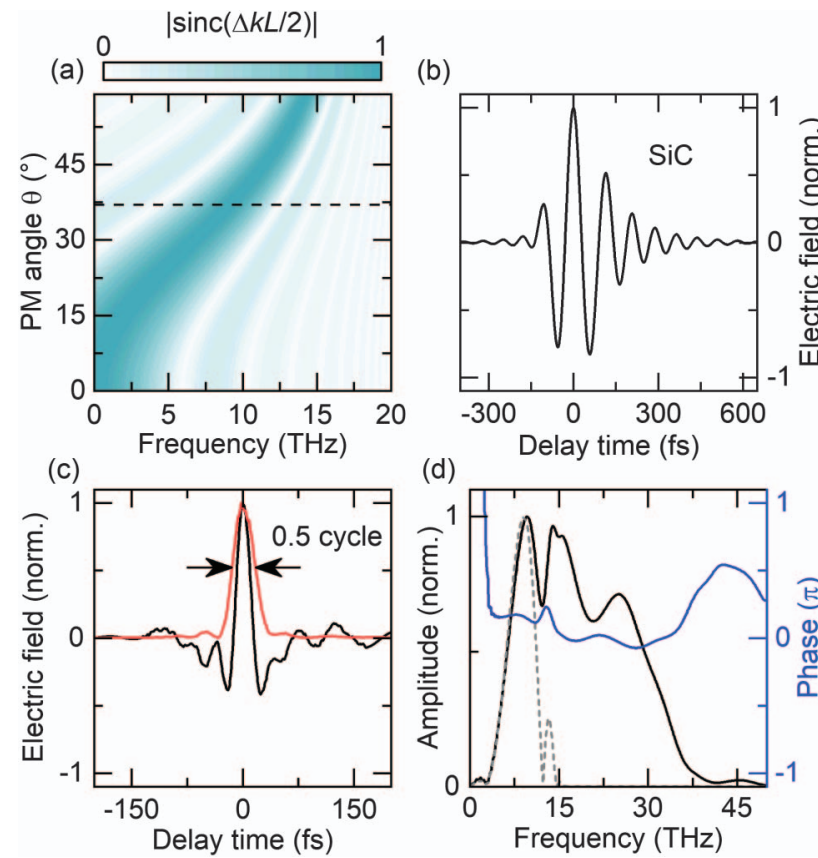

(d)

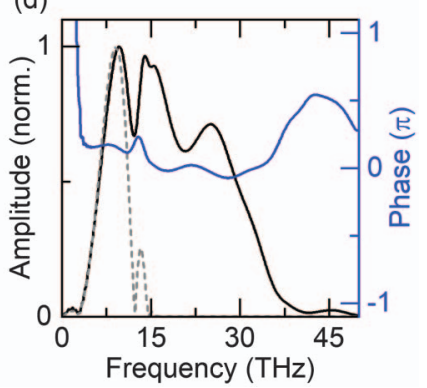

Fig. 4. Extending the bandwidth towards half-cycle waveforms. (a) Calculated PM function $|\operatorname{sinc}(\Delta k L / 2)|$ (color coded) as function of the PM angle and the idler frequency for type-I DFG in a $100 \mu \mathrm{m}$ thick $4 \mathrm{H}-\mathrm{SiC}$ crystal $\left(\lambda_{p}=790 \mathrm{~nm}\right)$. (b) Simulated waveform for optical rectification of the laser pulse in this $4 \mathrm{H}-\mathrm{SiC}$ crystal with type-I phase matching at $\theta_{\mathrm{SiC}}=37^{\circ}$ [see black dashed line in (a)]. (c) Simulated synthesized waveform (black curve) by temporal superposition of the field transients in Figs. 3(e) and 4(b). The pulse features 0.5 cycles within the FWHM of its intensity envelope (red curve). (d) Normalized spectral amplitude (black curve) and phase (blue curve) obtained by Fourier transforming the waveform in (c). The gray dashed line shows the spectral amplitude of the waveform in (b). 
of GaSe [see Fig. 2(c)]. To explore further expansion of the bandwidth, we simulate the coherent superposition of a third low-frequency multi-THz pulse. $4 \mathrm{H}-\mathrm{SiC}$ has been shown to work as an excellent medium for DFG between 5 and $15 \mathrm{THz}$ [27], smoothly extending the measured spectrum of Fig. 3(f) below $8 \mathrm{THz}$. Figure 4(a) displays the type-I phasematching function for a $100 \mu \mathrm{m}$ thick crystal of $4 \mathrm{H}-\mathrm{SiC}$. At an internal propagation angle of $\theta_{\mathrm{SiC}}=37^{\circ}$ with respect to the $Z$ axis [Fig. 4(a), black dashed line], the phase-matched frequency window gaplessly complements the amplitude spectrum of the synthesized subcycle pulse of Fig. 3(e). The numerically simulated electric field waveform generated in the $4 \mathrm{H}-\mathrm{SiC}$ crystal $\left(\theta_{\mathrm{SiC}}=37^{\circ}\right)$ with a flat spectral phase of the driving pulse is depicted in Fig. 4(b). When superimposing the transients featured in Figs. 3(e) and 4(b) with optimal delay time and relative amplitudes, the resulting waveform [Fig. 4(c), black curve] exhibits a single intense field spike, which exceeds the amplitude of all other local extrema within the waveform by a factor of 2.5 . The pulse duration of $30 \mathrm{fs}$ at a center frequency of $18 \mathrm{THz}$ corresponds to as few as 0.5 cycles under the intensity envelope [Fig. 4(c), red curve]. The amplitude spectrum generated in $4 \mathrm{H}-$ $\mathrm{SiC}$ [Fig. 4(d), gray dashed curve] complements the experimental result of Fig. 3(f) to yield a total spectrum extending over 2.3 optical octaves (FWHM), from 6 to $30 \mathrm{THz}$.

Field transients as shown in Figs. 3(e) and 4(c) could find immediate applications as ultrabroadband probe pulses. Plasmonic near-field enhancement could also make them valuable driving fields for strong-field control, such as light-wave-STM, where strictly CEP-stable, subcycle waveforms are required. In combination with modern high-power solid-state laser amplifiers, the field strengths could be scaled up to the $\mathrm{MV} / \mathrm{cm}$ range, which would also open exciting applications in solid-based highharmonic and attosecond generation.

In conclusion, the combination of optical rectification of multiple group velocity matched mixing stages with coherent pulse synthesis enables passively CEP-locked 0.8 -cycle pulses to be generated at a center frequency of $21 \mathrm{THz}$. In a first demonstration experiment, we achieved peak fields of $15 \mathrm{kV} / \mathrm{cm}$ at a repetition rate of $0.4 \mathrm{MHz}$ and fully characterized the electric field waveforms by EOS. Extending this concept to three-branch multiplexing, the pulse duration could be reduced to the halfcycle regime. We expect this all-parametric scheme to be widely scalable in pulse energy and repetition rate. Furthermore, the multiplexing idea could be spun towards yet more MIR branches using group velocity matched optical rectification in suitable nonlinear media. In order to optimize the overall conversion efficiency, simple beam splitter cubes for the laser output could be replaced with dichroic beam splitters, which select the optimal laser spectral components needed for the target difference frequency generated in each branch. This strategy would also add more sophisticated means of pulse shaping and offer exciting new perspectives for light wave electronics and resonant quantum control of condensed matter.

Funding. Deutsche Forschungsgemeinschaft (SFB 1277 (Project A05), SFB 1277 (Project B02)).

Acknowledgment. The authors thank Martin Furthmeier and Ignaz Laepple for technical assistance.
Disclosures. The authors declare no conflicts of interest.

\section{REFERENCES}

1. P. U. Jepsen, D. G. Cooke, and M. Koch, Laser Photon. Rev. 5, 124 (2011).

2. R. Ulbricht, E. Hendry, J. Shan, T. F. Heinz, and M. Bonn, Rev. Mod. Phys. 83, 543 (2011).

3. K. Liu, J. Xu, and X.-C. Zhang, Appl. Phys. Lett. 85, 863 (2004).

4. C. Kübler, R. Huber, S. Tübel, and A. Leitenstorfer, Appl. Phys. Lett. 85, 3360 (2004).

5. A. Sell, R. Scheu, A. Leitenstorfer, and R. Huber, Appl. Phys. Lett. 93, 251107 (2008).

6. M. Porer, J. Ménard, and R. Huber, Opt. Lett. 39, 2435 (2014).

7. S. Keiber, S. Sederberg, A. Schwarz, M. Trubetskov, V. Pervak, F. Kraus, and N. Karpowicz, Nat. Photonics 10, 159 (2016).

8. M. Knorr, P. Steinleitner, J. Raab, I. Gronwald, P. Merkl, C. Lange, and R. Huber, Opt. Express 26, 19059 (2018).

9. P. Gaal, W. Kuehn, K. Reimann, M. Woerner, T. Elsaesser, and R Hey, Nature 450, 1210 (2007).

10. G. Herink, D. R. Solli, M. Gulde, and C. Ropers, Nature 483, 190 (2012).

11. O. Schubert, M. Hohenleutner, F. Langer, B. Urbanek, C. Lange, U. Huttner, D. Golde, T. Meier, M. Kira, S. W. Koch, and R. Huber, Nat. Photonics 8, 119 (2014).

12. M. Hohenleutner, F. Langer, O. Schubert, M. Knorr, U. Huttner, S. W. Koch, M. Kira, and R. Huber, Nature 523, 572 (2015).

13. F. Langer, M. Hohenleutner, C. P. Schmid, C. Poellmann, P. Nagler, T. Korn, C. Schüller, M. S. Sherwin, U. Huttner, J. T. Steiner, S. W. Koch, M. Kira, and R. Huber, Nature 533, 225 (2016).

14. C. Schmidt, J. Bühler, A.-C. Heinrich, J. Allerbeck, R. Podzimski, D. Berghoff, T. Meier, W. G. Schmidt, C. Reichl, W. Wegscheider, D. Brida, and A. Leitenstorfer, Nat. Commun. 9, 2890 (2018).

15. T. L. Cocker, V. Jelic, M. Gupta, S. J. Molesky, J. A. J. Burgess, G. De Los Reyes, L. V. Titova, Y. Y. Tsui, M. R. Freeman, and F. A. Hegmann, Nat. Photonics 7, 620 (2013).

16. K. Yoshioka, I. Katayama, Y. Minami, M. Kitajima, and S. Yoshida Nat. Photonics 10, 762 (2016).

17. T. L. Cocker, D. Peller, P. Yu, J. Repp, and R. Huber, Nature 539, 263 (2016).

18. V. Jelic, K. Iwaszczuk, P. H. Nguyen, C. Rathje, G. J. Hornig, H. M. Sharum, J. R. Hoffman, M. R. Freeman, and F. A. Hegmann, Nat. Phys. 13, 591 (2017).

19. F. Junginger, A. Sell, O. Schubert, B. Mayer, D. Brida, M. Marangoni, G. Cerullo, A. Leitenstorfer, and R. Huber, Opt. Lett. 35, 2645 (2010).

20. T. P. Butler, D. Gerz, C. Hofer, J. Xu, C. Gaida, T. Heuermann, M. Gebhardt, L. Vamos, W. Schweinberger, J. A. Gessner, T. Siefke, M. Heusinger, U. Zeitner, A. Apolonski, N. Karpowicz, J. Limpert, F. Krausz, and I. Pupeza, Opt. Lett. 44, 1730 (2019).

21. Y. Nomura, H. Shirai, K. Ishii, N. Tsurumachi, A. A. Voronin, A. M. Zheltikov, and T. Fuji, Opt. Express 20, 24741 (2012).

22. E. A. Stepanov, A. A. Lanin, A. A. Voronin, A. B. Fedotov, and A. M. Zheltikov, Phys. Rev. Lett. 117, 043901 (2016).

23. P. Krogen, H. Suchowski, H. Liang, N. Flemens, K. Hong, F. X. Kärtner, and J. Moses, Nat. Photonics 11, 222 (2017).

24. A. Wirth, M. Th. Hassan, I. Grguras, J. Gagnon, A. Moulet, T. T. Luu, S. Pabst, R. Santra, Z. A. Alhamed, A. M. Azzeer, V. S. Yakovlev, V. Pervak, F. Krausz, and E. Goulielmakis, Science 334, 195 (2011).

25. C. Manzoni, O. D. Mücke, G. Cirmi, S. Fang, J. Moses, S. Huang, K. Hong, G. Cerullo, and F. X. Kärtner, Laser Photon. Rev. 9, 129 (2015).

26. H. Liang, P. Krogen, Z. Wang, H. Park, T. Kroh, K. Zawilski, P. Schunemann, J. Moses, L. F. DiMauro, F. X. Kärtner, and K. Hong, Nat. Commun. 8, 1 (2017).

27. M. Fischer, J. Bühler, G. Fitzky, T. Kurihara, S. Eggert, A. Leitenstorfer, and D. Brida, Opt. Lett. 42, 2687 (2017). 